\title{
Mate availability in small populations of plant species with homomorphic sporophytic self- incompatibility
}

\author{
DIANE L. BYERS \& THOMAS R. MEAGHER \\ Department of Biological Sciences, Rutgers University, PO Box 1059, Nelson Laboratories, Piscataway, New Jersey \\ 08855 USA
}

\begin{abstract}
Plants of a self-incompatible species, which occur in small populations, may have reduced fitness due to the limited availability of compatible mates. Self-incompatibility decreases inbreeding by allowing successful mating to occur only with individuals which differ by at least one-allele at the S-locus. A computer simulation model was developed to test the effect of small population size upon the diversity and the relative frequency of the S-alleles which determine the number of available mates. In a large population at equilibrium, the greater the number of $S$-alleles the greater the frequency of available mates for all individuals in the population. In small populations (less than 50 individuals), they are unable to maintain a high diversity of S-alleles and therefore there is a decrease in the frequency of available mates. In addition, in small populations there is an increase in the variance of available mates. The number of mates in these populations depends on the genotype of a particular individual. Two patterns would be expected in a small population of incompatible species: (1) a lower seed set per individual due to limited mates, and (2) an increase in variation of seed set among individuals due to the variance in available mates. Lower seed set would lead to a decrease in fitness of particular genotypes and could increase the possibility of local extinction of the species.
\end{abstract}

Keywords: allelic diversity, breeding systems, rare species, self-incompatibility, small populations.

\section{Introduction}

The rate of species extinction today is significantly greater than historical rates mainly due to habitat fragmentation and destruction (Wilson, 1988). As the number of rare species increases, interest in preservation of these species has also increased. Consequently, there has been significant effort focused on the determination of viable population sizes (Soule, 1987). The breeding systems of plants impose particular constraints on the mating structure, which needs to be considered in determining a viable population size. One type of breeding system which limits available mates in plants is self-incompatibility.

Self-incompatibility decreases inbreeding by limiting mating only to individuals which differ by at least one allele at the S-locus (De Nettancourt, 1977). The specific type of self-incompatibility determines the particular details of the incompatibility interactions. Homomorphic sporophytic self-incompatibility, in

Correspondence: D. L. Byers. which incompatibility is determined by an interaction between the diploid stigma and proteins originating from the diploid parent of the pollen grain, is considered in this paper. Homomorphic sporophytic selfincompatibility is widespread in both the Compositae and Brassicaceae (Richards, 1986). More recently, homomorphic sporophytic incompatibility has been found in species in the Convolvulaceae, Betulaceae, Caryophyllaceae and Sterculiaceae (Martin, 1968; Lindkvist, 1979; Thompson, 1979; Kowyama, et al., 1980).

Self-incompatibility may have evolved to avoid negative effects associated with inbreeding in plants (De Nettancourt, 1977). In small populations, where the availability of mates is limited by population size, self-incompatibility further limits the number of mates due to the constraints of the breeding system. Allele frequency variation among populations increases and diversity within populations decreases in small populations due to the effect of genetic drift (Wright, 1969). As individuals of self-incompatible species can only 
successfully mate with other individuals which differ for at least one S-allele, the level of allelic diversity will directly affect the number of mates available for each genotype in a population. Relative frequency of alleles will also influence mate availability. Individuals of a more common genotype would have less compatible mates than individuals of a rare genotype (assuming no dominance). Reduced seed set in small populations could result from the low number of available mates. Very limited numbers of available mates would have direct consequences for the fitness of a species. Most species occur in populations which are relatively large, therefore there is presumably sufficient allelic diversity so that the number of mates does not limit the seed set.

In two cases where the population size was small, the number of alleles has been estimated. Oenothera organensis, a rare species with gametophytic selfincompatibility, was found to have a large number of alleles (45 in a population of 500-1000) at the S-locus (Emerson, 1940). Both mutation and partial isolation of populations have been proposed to maintain this very high allelic diversity relative to the population size (Wright, 1960, 1964; Mayo, 1966). More recently, the Oenothera population has been found to contain about 5,000 individuals with no new estimates on the number of S-alleles (Levin et al. 1979). Carthamus flavescens, a weed that occurs in small populations, has a sporophytic breeding system and low allelic diversity in populations (Imrie \& Knowles, 1971).

The present study is part of a broader comparative investigation of the rare species Eupatorium resinosum (Compositae), and its closely related but much more common congener $E$. perfoliatum, to address various ecological and genetic hypotheses concerning rarity. Sullivan (1972) conducted a study of the diploid and polyploid species of Eupatorium in North America, and found evidence of self-incompatibility in all of the diploid species studied, including $E$. perfoliatum. Therefore it seems likely that $E$. resinosum, also diploid, is self-incompatible. We are presently performing a series of crosses to test this possibility directly. We have observed that in small populations $15-50$ flowering individuals) of $E$. resinosum the seed set of individuals was significantly lower than in larger populations (over 800 flowering individuals). It therefore seems likely that self-incompatibility may have a negative impact on the reproductive success in small populations of $E$. resinosum.

This paper examines the general relationship between population size and loss of allelic diversity at the S-locus and the consequences upon availability of mates. To address this issue a computer simulation model of mating events was used. The model explores the following situations.
1 The relationship between the number of $S$-alleles in the population and the number of available mates.

2 How the dominance structure among alleles effects the eqilibrium allele frequencies and the number of generations to reach equilibrium.

3 The rate of loss of alleles at the S-locus in small (finite) populations considering both overlapping and non-overlapping generations.

\section{Materials and methods}

A computer simulation model of mating events was used to test the effect of small population size on the availability of mates for a self-incompatible species. First the basic model is introduced, which assumes a large population size, to determine both the equilibrium allele frequencies and the number of available mates given a particular number of alleles. Then mating in a small population is simulated considering both overlapping and non-overlapping generations, examining loss of allelic diversity and its effect upon mate availability.

Sporophytic incompatibility is typically characterized by having a hierarchical dominance relationship among the S-alleles (De Nettancourt, 1977; Richards, 1986). Clearly, in a large population with dozens of $\mathrm{S}$-alleles the interactions among the alleles are potentially much more complex than a straightforward dominance heirarchy. However, we are addressing the consequences of allelic variation in small populations with very limited variation at the incompatibility locus, so that the effects of such dominance hierarchies are likely to play an important role in population dynamics. For the purpose of our simulation, we assumed a dominance hierarchy of $\mathrm{S} 1>\mathrm{S} 2>\mathrm{S} 3>\ldots \mathrm{S} n$, resulting in the compatibility relationships illustrated in Table 1 . This relationship of hierarchical dominance was used to determine compatibility during the computer simulations in the present model. Simulations were conducted in APL on an IBM personal computer using version 9.0 of the STSC APL/Plus interpreter.

The basic model assumes random mating, a large population size and non-overlapping generations. The simulation of mating was achieved by starting with a parental array of genotype frequencies at the S-locus. The model was designed such that any number (at any frequency) of initial S-alleles could be used. All combinations of the parental array, limited only by their compatibility, were produced to simulate a mating event. This method produced a new array of genotypic frequencies (progeny array) after one generation of mating. To determine the equilibrium allele frequencies the model was run until the genotype frequencies were equal (to the fourth decimal place) in two sequential 
Table 1 Mating compatibilities under sporophytic incompatibility. Allele dominance $S_{1}>S_{2}>S_{3}$

\begin{tabular}{|c|c|c|c|c|c|}
\hline & \multicolumn{5}{|l|}{ Male } \\
\hline & $\mathrm{S}_{1} \mathrm{~S}_{2}$ & $\mathrm{~S}_{1} \mathrm{~S}_{3}$ & $\mathrm{~S}_{2} \mathrm{~S}_{2}$ & $\mathrm{~S}_{2} \mathrm{~S}_{3}$ & $\mathrm{~S}_{3} \mathrm{~S}_{3}$ \\
\hline \multicolumn{6}{|l|}{ Female } \\
\hline $\mathrm{S}_{1} \mathrm{~S}_{2}$ & - & - & + & + & + \\
\hline $\mathrm{S}_{1} \mathrm{~S}_{3}$ & - & - & + & + & + \\
\hline $\mathrm{S}_{2} \mathrm{~S}_{2}$ & + & + & - & - & + \\
\hline $\mathrm{S}_{2} \mathrm{~S}_{3}$ & + & + & - & - & + \\
\hline $\mathrm{S}_{3} \mathrm{~S}_{3}$ & + & + & + & + & - \\
\hline
\end{tabular}

Not compatible $=-$

Compatible $=+$.

generations. This basic model is deterministic. We introduced stochastic effects when the basic model was modified for small population size. In the runs of the model to equilibrium, the starting allele frequencies were either the most dominant allele with the highest frequency $(0.8)$ and others being equal, or the most recessive allele with the highest frequency $(0.8)$ and the others being equal. The average frequency of available mates was determined by a weighted (by genotype frequency) average of compatible genotypes for each genotype.

The basic model was modified to consider overlapping generations. The mating was simulated as described above but the new genotypic frequencies are a combination of 40 per cent of the genotype frequencies from the previous generation and 60 per cent of the genotype frequencies resulting from the most recent mating event. This modification creates a 'memory' of the genotype frequencies of the past generation for the present generation.

To consider small populations, the basic model was modified by randomly choosing a set number $(10,25$, 30 or 50 ) of individuals from the array of individuals with the initial genotype frequencies before mating.
Only this set number of individuals was allowed to mate. This process used the pseudo-random number generator built into APL. Small populations were simulated for both overlapping and non-overlapping generations. Overlapping generations were determined by a combination of the genotype frequencies of 40 per cent from the previous generation (after drift) and 60 per cent resulting from the most recent mating event with the limited population size. The starting genotype frequencies used were the equilibrium frequencies (Table 2). All runs of small populations were repeated three times.

\section{Results}

When the population is in equilibrium (stable allele frequencies) the number of available mates is maximized for all genotypes in a large population. Hierarchical dominance results in a skewed frequency of the $\mathrm{S}$-alleles at equilibrium with the most recessive allele being the most frequent and the most dominant being the least frequent (Table 2). The number of generations to reach equilibrium increases as the number of alleles at the S-locus increases (Table 3). The variation in the numbers of generations to equilibrium is also affected by initial genotype frequencies. When the most dominant allele (S1) was the most frequent it took a greater number of generations to reach equilibrium. When the most recessive allele $(S n)$ was the most frequent it took a smaller number of generations to reach equilibrium. Overlapping generations produced the same patterns but generally took longer to reach equilibrium (Table 3 ).

The number of compatible mates increases as the number of alleles increases in a population which is in equilibrium. When there are at least nine alleles at the S-locus, individuals on average have a high frequency of compatible mates (Table 3 ).

The loss of alleles at the S-locus directly affects the availability of compatible mates. In small populations

Table 2 Equilibrium allele frequencies

\begin{tabular}{|c|c|c|c|c|c|c|c|c|c|}
\hline \multirow{2}{*}{$\begin{array}{l}\text { Number of } \\
\text { alleles }\end{array}$} & \multicolumn{9}{|c|}{ Frequencies } \\
\hline & $S_{1}$ & $\mathrm{~S}_{2}$ & $\mathrm{~S}_{3}$ & $\mathrm{~S}_{4}$ & $\mathrm{~S}_{5}$ & $S_{6}$ & $\mathrm{~S}_{7}$ & $\mathrm{~S}_{8}$ & $\mathrm{~S}_{9}$ \\
\hline 3 & 0.17 & 0.22 & 0.61 & - & - & - & - & - & - \\
\hline 4 & 0.13 & 0.15 & 0.20 & 0.52 & - & - & - & - & - \\
\hline 5 & 0.10 & 0.11 & 0.14 & 0.18 & 0.47 & - & - & - & - \\
\hline 6 & 0.08 & 0.09 & 0.12 & 0.13 & 0.17 & 0.42 & - & - & - \\
\hline 7 & 0.07 & 0.08 & 0.09 & 0.10 & 0.12 & 0.15 & 0.40 & - & - \\
\hline 8 & 0.06 & 0.07 & 0.07 & 0.08 & 0.09 & 0.11 & 0.16 & 0.35 & - \\
\hline 9 & 0.06 & 0.06 & 0.06 & 0.07 & 0.08 & 0.09 & 0.11 & 0.15 & 0.33 \\
\hline
\end{tabular}


for both overlapping and non-overlapping generations only four or five alleles were maintained with a population size of 50 (Table 4). As the population size decreased the number of alleles maintained for 50 generations also decreased. There was no difference in the loss of allelic diversity between overlapping and nonoverlapping generations in small populations.

Simulations of small populations (25 individuals) examined the generation-to-generation flux in compatible mates (Table 5). The average frequency of compatible mates slightly decreased with the smaller population size ( 25 compared to infinite). The variance among genotypes for compatible mates increased greatly immediately after the population size decreased and differed among generations. The simulations with six or nine alleles lost one allele within three generations.

\section{Discussion}

The simulations of the small populations showed their inability to maintain large numbers of alleles at the S-locus. In sporophytic incompatible species, the dominance structure among the alleles caused the more dominant alleles to be less frequent at equilibrium. This distribution of allele frequencies was also found in other studies which modelled sporophytic incompatibility (Imrie et al., 1972; Sampson, 1974; Charlesworth, 1988). These less frequent alleles may be easily lost with a decrease in population size due to genetic drift, which will decrease the number of available mates. In one-locus gametophytic systems there is no dominance among the S-alleles, so that the frequencies of the alleles are expected to be approximately equal at equilibrium (Richards, 1986). In these gametophytic systems alleles may be lost more slowly since selection does not bring about a skewed frequency distribution.

The hierarchical dominance typically found in sporophytic species increases the number of available mates relative to that produced by no dominance, by allowing an increased number of possible combinations of alleles to be compatible. In studies of the allelic interactions at the S-locus of sporophytic incompatible species, these interactions can be more complex than presented in this model. For example, the alleles in the

Table 3 The number of generations to equilibrium considering both nonoverlapping and overlapping generations

\begin{tabular}{llll}
\hline $\begin{array}{l}\text { Number of } \\
\text { available } \\
\text { alleles }\end{array}$ & $\begin{array}{l}\text { Number of } \\
\text { generations } \\
\text { (non-overlapping) }\end{array}$ & $\begin{array}{l}\text { Number of } \\
\text { generations } \\
\text { (overlapping) }\end{array}$ & $\begin{array}{l}\text { Frequency } \\
\text { of } \\
\text { mates }\end{array}$ \\
\hline 3 & $18-20$ & $15-27$ & 0.6667 \\
4 & $19-28$ & $24-46$ & 0.7503 \\
5 & $25-48$ & $37-52$ & 0.8001 \\
6 & $26-59$ & $37-66$ & 0.8334 \\
7 & $33-60$ & $36-79$ & 0.8572 \\
8 & $33-62$ & $53-97$ & 0.8748 \\
9 & $40-74$ & $59-77$ & 0.8887 \\
\hline
\end{tabular}

Table 4 The number of alleles lost in relation to population size. The model was a simulation for 50 generations

\begin{tabular}{|c|c|c|c|c|c|c|}
\hline \multirow{3}{*}{$\begin{array}{l}\text { Initial } \\
\text { number of } \\
\text { alleles }\end{array}$} & \multicolumn{6}{|l|}{ Population size } \\
\hline & \multicolumn{2}{|l|}{10} & \multicolumn{2}{|l|}{30} & \multicolumn{2}{|l|}{50} \\
\hline & Non-overlapping & Overlapping & Non-overlapping & Overlapping & Non-overlapping & Overlapping \\
\hline 3 & 1 & 1 & 0 & 0 & 0 & 0 \\
\hline 4 & $1-2$ & $1-2$ & $0-1$ & $0-1$ & 0 & $0-1$ \\
\hline 5 & 3 & $2-3$ & 1 & $1-2$ & 0 & 0 \\
\hline 6 & $3-4$ & 4 & $1-2$ & $2-4$ & 1 & $1-2$ \\
\hline 9 & 7 & 7 & 5 & $4-5$ & 3 & $4-5$ \\
\hline
\end{tabular}


Table 5 Generation to generation variance in mate availability with non-overlapping generations

\begin{tabular}{llll}
\hline $\begin{array}{l}\text { Number of } \\
\text { alleles }\end{array}$ & Generation & $\begin{array}{l}\text { Average } \\
\text { compatible mates }\end{array}$ & $\begin{array}{l}\text { Variance of } \\
\text { compatible mates }\end{array}$ \\
\hline 3 & 0 & 0.6667 & $7.40 \times 10^{-7}$ \\
& 1 & 0.6601 & $2.51 \times 10^{-3}$ \\
& 2 & 0.6325 & $8.67 \times 10^{-3}$ \\
& 3 & 0.5815 & $1.12 \times 10^{-1}$ \\
6 & 4 & 0.6387 & $7.42 \times 10^{-3}$ \\
& 0 & 0.8334 & $6.75 \times 10^{-6}$ \\
& 1 & 0.8180 & $2.69 \times 10^{-3}$ \\
& 2 & 0.7737 & $4.62 \times 10^{-3}$ \\
& 3 & 0.7690 & $1.17 \times 10^{-1}$ \\
& Lost one allele & & \\
& 4 & 0.7712 & $5.08 \times 10^{-3}$ \\
& 0 & 0.8887 & $2.03 \times 10^{-5}$ \\
& Lost one allele & & $1.14 \times 10^{-3}$ \\
& 1 & 0.8657 & $1.14 \times 10^{-3}$ \\
& 3 & 0.8662 & $2.06 \times 10^{-3}$ \\
& 4 & 0.8452 &
\end{tabular}

cells of the stigma may act independently while the alleles associated with the pollen have the dominance hierarchy (Richards, 1986). In all cases the equilibrium allele frequencies are not equal and the number of mates are increased due to the more complex interactions associated with the dominance hierarchy.

Considering the large number of generations it takes to reach equilibrium, only populations which are very large for a long period of time would be able to reach equilibrium (maximization of mate availability for all genotypes). Populations in which population size declines before reaching equilibrium will differ in the number of available mates for each genotype. Unless a population is in equilibrium some individuals with genotypes more frequent than their equilibrium frequencies will have a relatively low number of available mates. Other individuals with genotypes less frequent than their equilibrium frequencies will have a relatively high number of available mates. Therefore when a population is not at equilibrium, fitness (as measured by available mates) will vary considerably among the genotypes.

In simulations of small populations ( 25 individuals) the variance of available mates increased immediately after the population size decreased. This large variance in mate availability may be reflected in a population by the lower seed set of certain individuals whose genotypes are more common. Variance in mate availability may lead to loss of alleles which would further decrease the number of available mates. Mate availability quantifies the potential fitness of each genotype. Therefore variation in mate availability may be interpreted as variation in fitness among the genotypes.

The expected fitness patterns due to self-incompatibility in small populations are lower seed set and higher variance among plants in seed set compared to larger populations. Such patterns were observed in Eupatorium resinosum (D. L. Byers, personal observation).

Other studies have shown that limited pollinators' visits may, in small populations, result in a lower seed set (Jennersten, 1988). Limited pollinator attraction to small populations would interact with the limited number of S-alleles to further decrease reproduction of a population.

Demographic stochasticity has been considered a very important factor leading to local extinction of very small populations (Lande, 1988). If self-incompatibility in small populations leads to variation in seed set this could begin a downward cycle, and demographic stochasticity may accelerate possible extinction of populations. For example, in the summer of 1989, when New Jersey experienced unusually high rainfall, populations of $E$. resinosum in wet areas beside streams were submerged for days after each rain. Both the growth rate and flowering frequency were very low in these populations. This illustrates how environmental variation may decrease reproduction in popula- 
tions in some years, and when combined with self-incompatibility the resulting seed set may be very low.

Allelic diversity at loci other than the S-locus would be expected to be lost more quickly with small populations considering they are not necessarily selected to remain at a certain frequency like the S-alleles. The quick loss of any allelic diversity at loci not linked to the S-loci may increase mating of individuals which are genetically similar due to the loss of variation in the population.

Avoidance of inbreeding is proposed as an explanation for the evolution of self-incompatibility (De Nettancourt, 1977). In small populations self-incompatibility will prevent matings between close relatives, but as the degree of relatedness of the individuals is greater, the overall inbreeding level will be higher. Thus, possible negative effects of inbreeding could be seen due to the loss of genetic variation at the other loci. Studies to compare the level of genetic polymorphism of congeneric species, which differ in their breeding systems (self-compatible vs. self-incompatible), found the self-incompatible species to be more polymorphic (Solbrig, 1972; Gottlieb, 1973; Levin, 1978a: Ellstrand \& Levin, 1980). Oenothera organensis, a gametophytic self-incompatible species, had high allelic diversity at the S-locus but low diversity at other loci (Levin et al., 1979). O. organensis is a rare species which occurs in small populations. The small population size was considered the cause of the low diversity at most of the loci, while the frequency-dependent selection acting on the S-locus was considered to be the cause of this high diversity (Levin et al., 1979).

Various factors may maintain allelic diversity. Overlapping generations simulated in this model do not appear to affect the loss of S-alleles. In fact, the slower response of overlapping generations to reach equilibrium (maximization of compatible mates), due to its 'memory' of past generations, may keep the frequencies further from equilibrium and loss of alleles could occur more readily.

Seed banks consist of a mixture of seeds produced by many generations, so it may serve as a pool of genetic diversity (Levin, 1978b). A previous computer simulation model of sporophytic incompatibility (Imrie et al., 1972) examined the effects of both migration and seed banks in the preservation of S-allelic diversity. Imrie et al. (1972) showed that migration can help slow down the rate of loss of allelic diversity at the S-locus. The presence of a seed bank did not significantly affect the rate of loss of alleles.

Mutation was examined in various mathematical approximations and simulations to explain the high diversity of S-alleles in small populations of Oenothera organensis (Wright, 1960; Mayo, 1966; and others). These models mostly proposed different mutation rates that were necessary to maintain the S-allelic diversity found in this population. The population sizes in these studies were much larger (250) than the population size used in this model. Mutation rates required to maintain allelic diversity in the smaller populations considered here would have to be much higher than those reported elsewhere.

Both random mating and a homogeneous habitat were assumed in the model. Non-random pollen dispersal and a heterogenous habitat may substructure a population, such that the genotypes are locally adapted (Levin, 1978b). The allelic diversity at both the S-locus and the other loci may be maintained through these processes.

Why is self-incompatibility maintained in species that are commonly found in small populations? Selfcompatibility has been proposed to evolve from selfincompatibility in some species in small populations (Lloyd, 1965). This paper illustrates the disadvantage of self-incompatibility in small populations due to its negative impact on fitness. The maintenance of selfincompatibility in species occurring in a small population may be due to three possibilities: (1) no mutations of S-alleles to self-compatibility; (2) the disadvantages of inbreeding depression outweigh the disadvantage of low seed set; (3) the species is not at a demographic equilibrium and the populations over the long run will be large enough so self-incompatibility is favoured. If a mutation occurs which permits selfing it should rapidly increase provided inbreeding is not a major factor. Charlesworth (1988) showed that with significant inbreeding depression self-incompatible alleles could be maintained in a population with a self-compatible allele. Charlesworth's model only considered large populations but it illustrates the importance of inbreeding depression in the maintenance of selfincompatibility.

Very small populations of incompatible species, as illustrated by this model, are not stable. The breeding system will cause small populations to more likely become extinct due to variance and limited availability of mates.

\section{Acknowledgements}

We are indebted to the insightful comments of Dr S. Handel and C. Bristow on an earlier draft of this paper. This project was partially supported by the Charles and Joanna Busch Summer Fellowship of Rutgers University and a grant from Rutgers University Graduate School. 


\section{References}

CHARLESWORTH, D. 1988. Evolution of homomorphic sporophytic self-incompatibility. Heredity, 60, 445-453.

DE NETTANCOURT, D. 1977. Incompatability in Angiosperms. Springer-Verlag, Berlin.

ELLSTRAND, N. C. AND LEviN, D. A. 1980. Recombination system and population structure in Oenothera. Evolution, 34, 923-933.

EMERSON, S. 1940. Growth of incompatible pollen tubes in Oenothera organensis. Bot. Gaz., 101, 890-911.

GoTTLIEB, L, D. 1973. Genetic differentiation, sympatric speciation, and the origin of a diploid species of Stephanomeria. Am. J. Bot., 60, 545-553.

IMRIE, B. C., KIRKMAN, C. J. AND ROSS, D. R. 1972. Computer simulation of a sporophytic self-incompatibility breeding system. Aust. J. Biol. Sci., 25, 343-349.

IMRIE, B. C. AND KNOWLES, P. F. 1971. Genetic studies of selfincompatibility in Carthamus flavescens Spreng. Crop Sci., 11, 6-9.

Jennersten, o. 1988. Pollination in Dianthus deltoides (Caryophyllaceae): Effects of habitat fragmentation on visitation and seed set. Conserv. Biol., 2, 359-366.

KOWYAMA, Y., SHIMANO, N. AND KAWASI, T. 1980. Genetic analysis of incompatibility in the diploid species of Ipomoea closely related to the sweet potato. Theoret. Appl. Genet., 58, 149-155.

LANDE, R. 1988. Genetics and demography in biological conservation. Science, 241, 1455-1460.

LINDKvist, A. 1979. One-locus sporophytic self-incompatibility in the carnation family, Caryophyllaceae. Hereditas, 91, 307 (Abstract).

LEVIN, D. A. 1978a. Genetic variation in annual Phlox: selfcompatible species. Evolution, 32, 245-263.

LEVIN, D. A. $1978 \mathrm{~b}$. Some genetic consequences of being a plant. In: Brussard, P. F. (ed.) Ecological Genetics: The Interface, Springer-Verlag, New York, pp. 189-214.
LEVIN, D. A., RITTER, K., AND Ellstrand, N. C. 1979. Protein polymorphism in the narrow endemic Oenothera organensis. Evolution, 33, 534-543.

LLOYD, D. G. 1965. Evolution of self-compatibility and racial differentiation in Leavenworthia (Cruciferae). Contr. Gray Herb., 195, 3-134.

MARTIN, F. W. 1968. The system of self-incompatibility in Ipomoea. J. Hered., 59, 263-267.

MAYO, o. 1966. On the problem of self-incompatibility alleles. Biometrics, 22, 111-120.

Richards, A. J. 1986. Plant Breeding Systems, Allen and Unwin, London.

SAMPSON, D. R. 1974. Equilibrium frequencies of sporophytic self-incompatibility alleles. Can. J. Genet. Cytol., 16. 611-618.

SOLBRIG, O. T. 1972. Breeding system and genetic variation in Leavenworthia. Evolution, 26, 155-160.

soule, M. E. 1987. Viable Populations for Conservation, Cambridge University Press, Cambridge.

Sullivan, v. I. 1972. Investigations of the breeding systems, formation of auto- and alloploids on the reticulate pattern of hybridization in North American Eupatorium (Compositae). Ph. D. Thesis Florida State University, USA.

THOMPSON. M. M. 1979. Genetics of incompatibility in Corylus avellana L. Theoret. Appl. Genet., 54, 113-116.

WILSON, E. O. 1988. Biodiversity, National Academy Press, Washington D.C.

WRIGHT, s. 1960 . On the number of self-incompatibility alleles maintained in equilibrium by a given mutation rate in a population of given size: a reexamination. Biometrics, 16 , 61-85.

WRIGHT, s. 1964. The distribution of self-incompatibility alleles in populations. Evolution, 18, 609-619.

WRIGHT, s. 1969. Evolution and the Genetics of Populations. Vol. 2 The Theory of Gene Frequencies. University of Chicago Press, Chicago, IL. 